\title{
The Silence of Physics
}

\author{
Barry Dainton ${ }^{1}$
}

Received: 21 October 2020 / Accepted: 18 August 2021

(C) The Author(s) 2022, corrected publication 2022

\begin{abstract}
Although many find it hard to believe that every physical thing - no matter how simple or small-involves some form of consciousness, panpsychists offer the reassurance that their claims are perfectly compatible with everything physics has to say about the physical world. This is because although physics has a lot to say about causal and structural properties it has nothing to say about the intrinsic natures of physical things, and if physics is silent in this regard it is perfectly possible that everything physical has an experiential intrinsic nature. Following in Thomas Nagel's footsteps, panpsychists have also argued that by revealing that everything is composed of the same fundamental ingredients, physics provides grounds for holding that if any physical things (e.g. our neural processes) have an experiential intrinsic nature then all must. My main contention in this paper is that the relationship between physics and panpsychism is considerably more complex than panpsychists have tended to assume. Nagel's reasoning may be sound in the context of simplistic atomic theories which posit just one kind of fundamental particle. However, it begins to look distinctly dubious in the context of the diverse range of primitive entities that are to be found in the Standard Model of particle physics. Galen Strawson has suggested that mass-energy interconvertibility should be regarded as evidence that everything physical has the same intrinsic nature. I suggest Strawson's claim relies on a dubious construal of the nature of energy. Special relativity is another of the cornerstones of contemporary physics, and it too makes life difficult for panpsychists, a fact which emerges when we consider what it would like to be a ray of light. However in this case I suggest that there is an interesting-if radical-move open to the panpsychist: they can simply deny that light exists. To conclude I briefly consider whether what QCD has revealed about the nature of mass poses a problem for panpsychism.
\end{abstract}

Barry Dainton

bdainton@liverpool.ac.uk

1 Department of Philosophy, University of Liverpool, Liverpool L67 7ZY, UK

Published online: 09 November 2021 
"Hello darkness, my old friend."

\section{Introduction}

Interest in the venerable doctrine of panpsychism has increased considerably over the last decade or so. The claim that every physical thing, no matter how small and insignificant, possesses some form of conscious experience is no longer confined to eccentric animistic cults, it is also being taken increasingly seriously by a growing number of hard-headed analytic philosophers.

The main reason for this is straightforward. The various attempts to reduce experiential properties to properties of a fundamentally different kind (dispositions to behave, functional properties, neural properties, representational properties etc.) have all turned out to be problematic. Dualism, the main non-reductionist alternative, is also problematic-if consciousness is immaterial, how does it manage to interact with the physical? The interaction issue doesn't arise if consciousness is itself a physical phenomenon right at the heart of matter, as the panpsychists maintain. It's true that you won't find any mention of phenomenal properties such as colour and sound (construed as phenomenal properties) in physics and chemistry textbooks. However, panpsychists point out that this is only a problem if you also believe that the physical properties recognized by current physics and chemistry are all the physical properties there are, and there are good reasons for supposing this not to be the case. For one thing we know that our current science is incomplete: we don't yet have an adequate theory of quantum gravity, for example. No less importantly, physicists may do a good job of discovering the structural and causal properties of the fundamental ingredients of the physical world, but they make no attempt to describe the intrinsic or categorical natures of these ingredients. They tell us, for example, that an electron has a certain mass or size, but physics is entirely silent on the intrinsic nature of the stuff that constitutes electrons. Strawson makes the point thus:

Physics is magnificent: many of its claims are either straightforwardly true or very good approximations to truth. But all of its claims about the physical are expressed by statements of number or equations. They're truths about quantities and relational structures instantiated in concrete reality; and these truths tell us nothing at all about the ultimate nature of the stuff of reality, the stuff that has the structure that physics analyzes. ... This is the silence of physics ...

(Strawson, 2018)

Evidently if physics is silent in this manner, then when a panpsychist claims that all physical things have an experiential intrinsic nature this claim is not in conflict with anything physics has to say. ${ }^{2}$

\footnotetext{
${ }^{1}$ Paul Simon, "The Sound of Silence".

2 It should be noted that most contemporary panpsychists don't believe that everything is conscious in the same sort of way-thus avoiding the implausible result that chairs and tables are conscious subjects in the way we are.
} 
So there are reasons for taking panpsychism seriously, and this has long been recognized. If the doctrine has long held appeal for the mystically inclined its being taken seriously in more serious circles is by no means a new phenomenon either. At the start of the twentieth century the thesis that matter can possess an experiential (or quasi-experiential nature) was endorsed by philosophers such as Bergson, James, Drake and Russell, as well as by physicists such as Eddington.

Some earlier panpsychists - and James in particular-were much exercised by problems that contemporary panpsychists are now grappling with. Do small-scale physical things such as elementary particles or biological cells consist of unified centres of consciousness in their own right? If so, the panpsychist is confronted with a combination problem. Our own states and streams of consciousness are wholly constituted by neural states and processes (or so many panpsychists maintain), which are themselves wholly constituted by individual cells and particles. How do the elementary minds the latter possess manage to combine to form more complex states of consciousness, such as our own? $?^{3}$ Since it is not obvious that individual conscious subjects can combine in the required way some panpsychists have been tempted to reject a pluralistic ontology in favour of a monistic one: the entire material universe consists of just one all-encompassing conscious subject, a subject that we are all parts of. But if this "cosmo-panpsychism" avoids combinatorial problems, it gives rise to difficulties of its own. If your experiences and mine are both states of a single subject's consciousness it is puzzling that I am not aware of your experiences in the way I am aware of my experiences, since on this view we are one and the same.

There are moves panpsychists can make in response to these difficulties, but I am not going to comment further on them here. Instead I want to focus on difficulties of a rather different sort. In the century or so which separates contemporary panpsychists from James, Eddington and Bergson the picture of the physical world provided by physics has evolved very considerably. In many respects, thanks in large part to Einstein's relativity and the rise of quantum theory, the physicists' account of the nature of physical reality is an exceedingly strange one in several respects. However, in the recent debates little has been said about how these scientific developments impact upon the plausibility or otherwise of panpsychism. Given the complexity of the relevant sciences - not to mention the continuing interpretational controversies surrounding quantum theory - this is a huge topic, one to which I cannot begin to do justice here. Nonetheless, I think even the preliminary investigations which follow show that there are potentially interesting issues in this connection which panpsychists should not overlook.

\footnotetext{
3 This is a problem for so-called "constitutive" panpsychists, who hold that our own highly complex conscious states come into existence when elementary conscious constituents in our brains combine in certain ways. The problem doesn't arise for "emergentist" panpsychists who deny that high-level consciousness is composed of multitudes of elementary conscious entities-for more on this distinction see Goff (2017b) and Brüntrup (2016). Since in what follows we will be concerned with the rationale for supposing that the elementary ingredients of the physical world possess any form of consciousness, this distinction will not be relevant.
} 


\section{Getting the Pan- into Panpsychism}

I want to start by taking a step back and take a closer look at one of the core arguments propounded by panpsychists. We begin by adopting three views that are central to the case for panpsychism in its recent guises. First, we assume consciousness is a real and ineliminable component of reality. Second, we reject dualism in any of its guises. Third, we reject the claim that consciousness is an emergent high-level physical property, analogous to solidity or liquidity. With these assumptions in place it can seem very plausible to suppose that our experiences are identical with certain fundamental physical processes in our brains and nervous systems. As a consequence, given that our experiences possess intrinsic properties of a phenomenal kind, so too must the relevant physical processes in our brains and nervous systems. But given this limited starting point, what justifies the further claim that all physical processes everywhere in the universe-large or small, past, present and future-also have experiential intrinsic natures?

Russell was an early (and highly influential) proponent of the view that physics only provides us with causal and structural information about the physical world: "The aim of physics, consciously or unconsciously, has always been to discover what we may call the causal skeleton of the world." (Russell, 1927, p. 391) If physics is entirely silent with respect to the intrinsic natures of physical phenomena, there is no obstacle to supposing that certain physical processes in our brains have experiential inner natures - the relationship between consciousness and matter is no longer mysterious. But Russell was reluctant to take the further step and hold that all physical phenomena have an experiential intrinsic nature. He consistently regarded this additional step as unwarranted by the available evidence: "I do not myself believe that the philosophical arguments for the view that all reality must be mental are valid. But I also do not believe that any valid arguments against this view are to be derived from physics. The only legitimate attitude about the physical world seems to be one of complete agnosticism as regards all but its mathematical properties." (Russell, 1927, pp. 270-271). ${ }^{4}$

In the more recent literature there is an influential line of reasoning which does serve to put the "pan" into panpsychism, and justifies taking the extra step that Russell resisted. It derives from Nagel, and one of his presentations of it unfolds thus:

Our bodies and in particular our central nervous systems belong to that physical world, as do the bodies of all other organisms capable of mental activity. ... There is nothing unique in the physical composition of our bodies; only their chemical and physiological structure is unusual. An animal organism is composed of ordinary elements, which are in in turn composed of subatomic particles found throughout the known universe. A living human body can therefore be constructed out of a sufficient quantity of anything-books, bricks, gold,

\footnotetext{
${ }^{4}$ Russell was well aware that others-notably Eddington and Whitehead-were willing to draw this more radical conclusion, but he made it clear that he disapproved; see his 1928 review of Eddington's The Nature of the Physical World.
} 
peanut butter, a grand piano. The basic constituents just have to be suitably rearranged. The only way of actually producing such a rearrangement is by the natural biological process of nourishment and growth, but this does not alter the fact that the materials can come from anywhere. $(1986, \text { p. 28) })^{5}$

If our experiences are indeed identical with neural states and processes then we can be confident that the elementary physical constituents of at least these material processes possess phenomenal properties. But on a purely physical level, Nagel points out, there is nothing at all special about these neural processes: they are entirely composed of perfectly ordinary atoms, molecules and electro-chemical processesthere are no mysterious unknown ingredients, just ordinary physical stuff. Furthermore, there is nothing special about the elementary constituents that can be found in our brains: all compound physical entities are composed of the same basic ingredients. If the elementary constituents of our neural processes have intrinsic natures of a phenomenal kind we have little option but to conclude that the constituents of all physical things have experiential intrinsic natures, since they all are composed of the same elementary ingredients.

If we don't take this step, we are forced into the unpalatable position of maintaining that electrons, neutrinos and other elementary particles possess different intrinsic natures when they are participating in neural processes than they do on other occasions. Aside from the fact that his complicates the laws of nature to a significant degree, we also have to provide a plausible explanation as to how elementary particles manage to detect when they are participating in consciousness-involving physical processes and vary their intrinsic natures accordingly. Since this looks to be an uphill struggle - to put it mildly - it looks as though Russell's agnostic stance was unwarranted: there are powerful reasons for supposing that everything that physical has an experiential inner nature. ${ }^{6}$

\section{From Ancient Atomism to the Periodic Table}

Nagel's reasoning can seem persuasive on first encountering it-I certainly found it so. But how persuasive should we find it? Here much—everything, in fact-depends on what we take the basic ingredients of the physical world to be, and different views on this issue have very different implications.

Let's suppose you're a follower of the ancient atomist Democritus who subscribes to a particularly simple form of the atomic theory of matter. More specifically, you

\footnotetext{
5 Nagel emphasizes the same point on the opening page of his earlier "Panpsychism" (1979) "The matter composing us is not special; the matter composing any material entity, if broken down far enough and rearranged, could in principle be incorporated into a living organism.".

${ }^{6}$ It should be noted that this general line of argument by no means originated with Nagel, and some earlier panpsychists deployed it as well. In his Mind and its Place in Nature Drake argues that since we are built of the same constituents as other things "we are ... free to believe that the stuff that is deployed in this or that order throughout the universe is the same sort of stuff that composes us, sentient beings that we are" (1925, p. 100).
} 
hold that (a) everything in the physical world is composed of indivisible atoms of small but finite size, (b) there is just one type of atom, and (c) all atoms have the same categorical and dispositional properties. If this form of atomism is true, then Nagel's reasoning looks to be sound. If those physical processes in our brains which are identical with our experiences possess phenomenal properties, then so do the constituents of everything else. How could it be otherwise, since all physical things are made of atoms, and atoms are all exactly alike?

Now let's move on a couple of millennia and ponder how things stand at the start of the nineteenth century. Atomism received a considerable boost with the publication of John Dalton's A New System of Chemical Philosophy in 1808. A total of twenty basic elements were listed in the first edition; when the second edition was published in 1827 this number had grown to 36. The only aspect of Dalton's atomism relevant to current purposes is that he held that each element has its own kind of indivisible atom, each with a characteristic mass and pattern of behaviour. If there are 36 different elements there are 36 different types of atom.

As is obvious, Nagel's argument looks far less secure in the context of Daltonian atomism than it does in the case of the single kind of atom theory we considered initially. Subsequent research has uncovered additional forms of matter, and it is now thought that there are 92 naturally occurring elements on the Earth. If there are 92 different kinds of fundamental element it is far from absurd to suppose that only some elements figure in the neural processes which constitute conscious experiences. This is an eminently testable hypothesis which can be verified or falsified by tests conducted on brain autopsies. Not surprisingly a good deal of research has been done in this area, and contemporary biologists believe that only 25 or so of the 92 naturally occurring elements can commonly be found in human bodies (Fig. 1).

Fortunately for Nagel, as most of us will remember from our school days, chemistry has moved on since Dalton's day. As already noted, a sizable number of additional elements have been discovered. More significantly, although each different element is associated with a different kind of atom, these atoms are not the structureless fundamental entities that Dalton took them to be. According to the schoollevel chemistry picture, the atoms all have constituent parts in the guise of protons, electrons and neutrons. These different particles are akin to miniature planetary systems, with the electrons in orbit around the protons and neutrons which lie tightly clustered at centre of the atom. What differentiates the different atoms found in the periodic table is the possession of different numbers of protons, neutrons and electrons. Hydrogen has just one proton and one electron; carbon has six electrons and six protons, hafnium has seventy two of each. ${ }^{7}$

How does Nagel's argument fare in this more modern context? The situation is certainly a considerable improvement over the Daltonian picture: we are no longer dealing with dozens of different kinds of fundamental particle. But Nagel is by no

\footnotetext{
7 Rather muddying this simple picture is the fact that while elements invariably possess the same numbers of protons and electrons, the number of neutrons they possess can vary in hard-to-predict ways. Carbon has six protons and six neutrons, hafnium has 72 protons and 174 neutrons. But these additional complexities don't affect the basic picture: everything is made of the same three basic ingredients.
} 


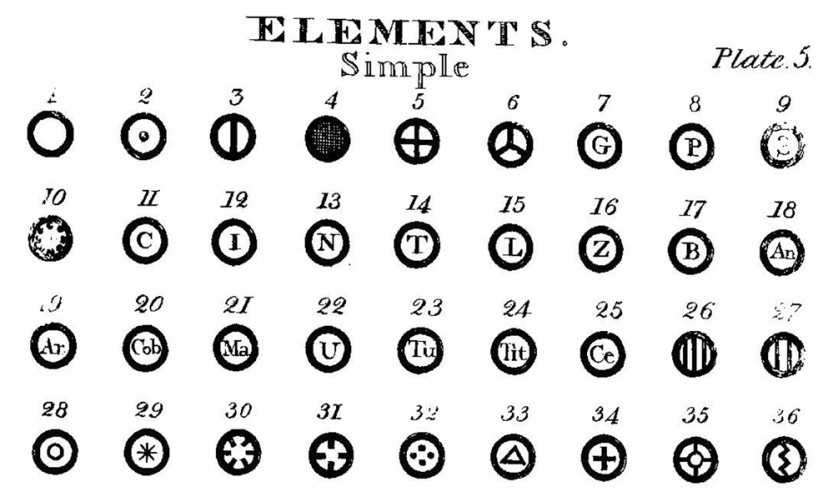

Fig. 1 The expanded 2nd edition version of Dalton's Periodic Table, where there is a different kind of fundamental particle for each of the 36 elements. (Dalton 2010, plate 5)

means entirely out of the woods. On the view in question electrons, protons and neutrons are three different kinds of elementary particle. Consequently, as in the Dalton scenario, it seems quite conceivable that some kinds of elementary particles have experiential intrinsic natures while others do not. Perhaps electrons possess a conscious inner life, but protons and neutrons don't. Or perhaps all and only electrically charged particles are conscious - in which case protons and electrons will possess consciousness, but neutrons won't. It may well be that there are good reasons for rejecting "hybrid" hypotheses of the latter kind, where different fundamental entities have very different kinds of intrinsic nature, but if so the panpsychist should spell out what these reasons are, rather than remaining silent on the issue.

\section{The Standard Model}

The familiar tri-partite conception of atomic structure emerged in the 1930's, with experiments which first revealed the existence of the neutron (protons had been discovered by Rutherford in 1908). Since then our understanding of the deep structure of matter has evolved considerably. The discovery of the neutron was swiftly followed by the discovery in subsequent decades of a great many other subatomic particles: positrons (and all the other "anti-particles"), muons, muon neutrinos, tau leptons, eta mesons, phi mesons, upsilon mesons, up quarks, down quarks, strange quarks, charmed quarks, pions, kaons, gluons, the recently discovered Higgs boson-to mention just a few of the hundreds of exotic particle types that are now known to exist. Some of these (e.g. the positron) were first posited for theoretical reasons - their existence could be deduced from the mathematical equations. Others were detected empirically, typically as products of collisions between other subatomic particles.

Have these more recent discoveries taken us back to the Dalton era so far as Nagel's argument is concerned? This would be the case if these hundreds of subatomic particles were all fundamental, but - fortunately for the panpsychist—on 


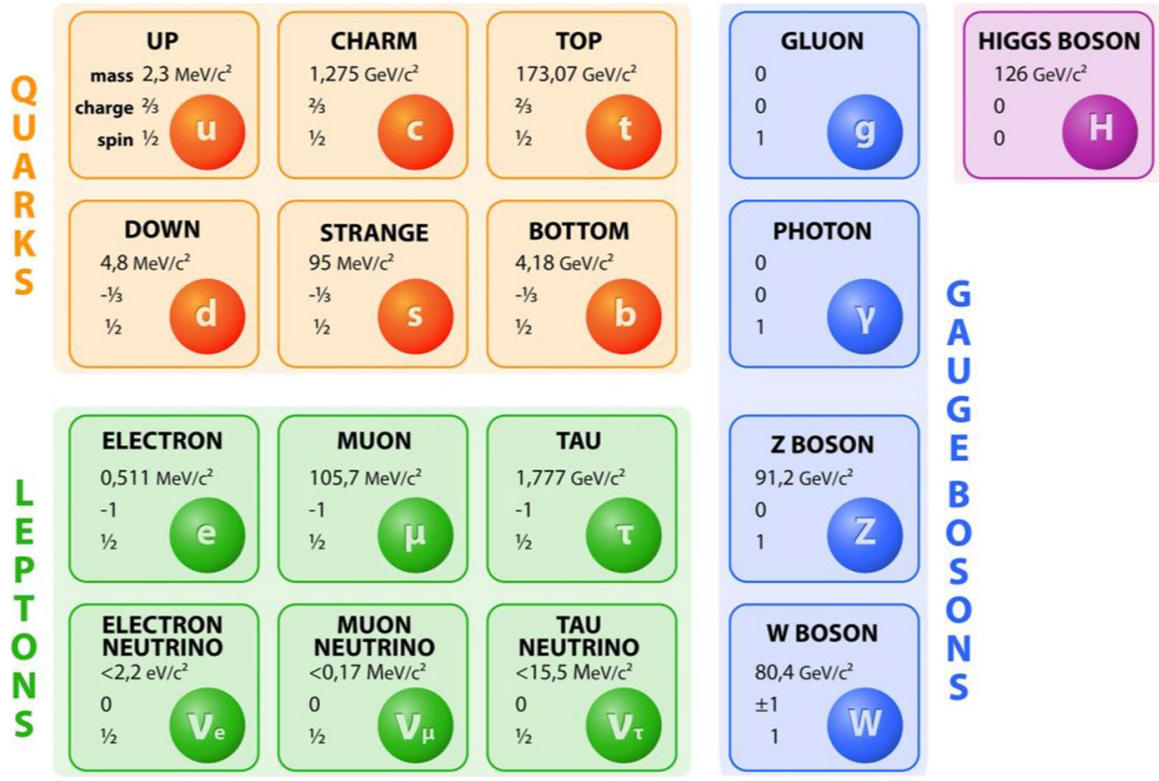

Fig. 2 The Standard Model's fundamental particles (Adobe Stock)

current thinking this by no means the case. According to the "Standard Model" of particle physics, painstakingly put together since the 1970's, there is a comparatively small number truly fundamental types of entity, and all the others are members of the subatomic zoo are composed of these. ${ }^{8}$

As can be seen in Fig. 2, the elementary particles of the Standard Model are typically arranged in different groups or families. The bosons over on the right are regarded as "force carrying" particles, with the W, Z particles \& gluons operating over (very) short distances within atomic nuclei and quarks, the photons and Higgs are not so confined. Over on the left we have the fermions, which fall into two categories: the six quarks (up/down/charm/strange/top/bottom) and the six leptons (electron, muon, tau, together with the electron neutrino, the muon neutrino and the tau neutrino). As well as more familiar properties such as mass and charge quantum

\footnotetext{
8 The Standard Model is a quantum field theory, and for most theorists this means the fundamental entities are universe-wide fields, and particles exist as localized mobile quanta of energy that only manifest when the fields are probed or measured in some way-rather than being the autonomous self-contained entities of classical atomism. Accordingly, in addition to the familiar electromagnetic field in the Standard Model there are also quark fields, electron fields, muon fields, gluon fields, W fields and neutrino fields - to mention but a few of those currently recognized. The issue of how quantum field theories should be themselves be interpreted is a controversial issue, and as things currently stand it is far from clear what we can conclude about the nature of reality from their remarkable empirical success. For a sample of relevant issues see Fraser (2009, 2011), Wallace (2011), and for an overview see Kuhlman (2020). However, as far as I can see my main claim in this section-that contemporary physics complicates matters for the panpsychist considerably-is largely independent of how these interesting debates end up being resolved.
} 
particles possess another basic quantum property: spin, which is analogous in some ways to angular momentum. Whereas the fermions all have half-integer spin $(1 / 2$, $3 / 2,5 / 2 \ldots)$ the bosons have integer spins $(0,1,2 \ldots)$. For each of the fermions there is a corresponding anti-particle possessing the same mass and spin but differing in other quantum properties such as charge and parity. If as is commonly (but not invariably) done we regard particles and anti-particles as instances of the same particle-type, we have a total of 17 fundamental particles.

So far as the quarks are concerned those classed as belonging to the second and third generations (i.e. the charm, strange, top and bottom) are extremely short-lived, and do not constitute stable forms of matter. The protons and neutrons which are found in ordinary atoms are composed exclusively of first generation up quarks and down quarks (protons consist of two ups and one down, neutrons are composed of two downs and one up). So far as the leptons are concerned, the electron is stable and long-lived and possesses mass and charge; the muon and tau are less stable but also possess mass and charge. The neutrinos, in contrast, possess no charge and negligible mass, and for this reason are very hard to detect-about 65 billion emanating from the sun are thought to pass through every centimeter of our bodies every second.

How does Nagel's reasoning stand up in the thoroughly contemporary context of the Standard Model? A dozen and a half different types of elementary entity is certainly not as problematic for the panpsychist as the ninety two that we would have to deal with if we conceived atoms in a Dalton-esque fashion. Even so, it is still a sizable number given that Nagel's argument is only unproblematic for conceptions of the physical world in which there is just a single type of atom with a uniform nature. Consequently it seems perfectly conceivable that only some of the types of elementary entities postulated by the Standard Model possess consciousness, whereas others do not. By way of illustration, we have just seen that only some of the Standard Model's fundamental ingredients routinely occur in ordinary forms of matter. Since the neural processes in our brains are composed exclusively of ordinary forms of matter, these processes will be constituted solely of electrons, up quarks and down quarks (the constituents of protons and neutrons), gluons and photons-the carriers of electromagnetic force and energy. If the more exotic quarks and neutrinos play no significant role in neural processes the panpsychist cannot straightforwardly conclude that all kinds of physical phenomena possess experiential intrinsic natures simply because some do. For contrary to what Nagel might have assumed, it's not the case that all the fundamental forms of matter exist in our brains and participate in neural processes. For the panpsychist's radical and far-reaching conclusion to be warranted additional considerations need to be brought into play.

\section{Homogeneity and Simplicity}

Recent proponents of panpsychism are aware that something needs to be said by way of a defense of the claim that everything physical has the same sort of categorical nature. In his influential "Realistic Monism: Why Physicalism Entails 
Panpsychism" (2006a) Strawson draws a distinction between "micropsychism", the doctrine that only some fundamental physical entities have an experiential nature, from "panpsychism", the claim that all the fundamental physical entities have an experiential nature. Strawson makes it clear that he finds micropsychism implausible: "I would bet a lot against there being such radical heterogeneity at the bottom of things." (2006a, p. 25) But he does not here elaborate on why he's willing to bet so heavily on the physical world's possessing a homogeneous intrinsic nature. Strawson fills this lacuna in a more recent article, where he suggests that "reasons of theoretical parsimony, simplicity and elegance tell in its favour ... given that some physical stuff is certainly experiential, [this] is the simplest hypothesis: all physical stuff is in its fundamental nature wholly experiential in all conditions and in all respects and all the way down." (Strawson, 2013) Calling it "The Simplicity Argument for Panpsychism" Philip Goff has defended a similar view:

Of all metaphysical theories consistent with the reality of ordinary consciousness, panpsychist forms of Russellian monism are the most simple, elegant and unified ... All we get from physics is this big black and white structure, which we metaphysicians must somehow colour in with concrete categorical nature. Assuming the falsity of substance dualism, we know how to colour in one bit of it: the brains of organisms are coloured in with consciousness. How to colour in the rest? The most elegant, simple, sensible option is to colour in the rest of the world with the same pen. (2017a, §7.2, pp. 169-171)

Since simplicity and unifying power are widely accepted as grounds for distinguishing between competing scientific theories, and since panpsychism is, after all, a theory about the nature of the physical world, it is by no means absurd to allow such considerations to play a role in our deliberations.

Simplicity and elegance generally enter the picture when we are trying to decide between two theories which are in other respects equally well-matched. Does the hypothesis that there is only one basic kind of categorical nature in fundamental physical entities (the experiential variety) fit the available data as well as the hypothesis that this is not the case? Strawson insists that it does:

The hypotheses (i) there is only experiential being, (ii) there exists both experiential and non-experiential being are empirically equivalent (theories are empirically equivalent if no empirical test can decide between them). This is because it seems that our best and most fundamental science-physics-can't decide between them? Why not? Because of the silence of physics on the question of the intrinsic structure-transcendent nature of reality. (2020, 332 with some of Strawson's numbering omitted)

If physics really were silent in the way Strawson suggests, then simplicity considerations might well favour hypothesis (i) over hypotheses (ii), and we would be well on the way to panpsychism. But is physics really silent? As we shall see, there are a number of ways in which physics might not be silent, and one of Strawson's own commitments provides one route to precisely this destination. 
Let us suppose that Strawson is right when he maintains that the relationship between an object's intrinsic or categorical nature and its causal powers is itself a necessary one. On Strawson's view, if an object is causally disposed to behave in certain ways in certain circumstances it is because it has a certain intrinsic nature. ${ }^{9}$ Mørch has also recently argued that panpsychists should adopt this view of the relationship between intrinsic natures and causation: "The phenomenal powers view is the view that phenomenal properties are intrinsically powerful, which is to say that they produce or bring about their effects, of make them happen, in virtue of their intrinsic character alone. Production should be understood as a defeasible necessary connection ..." $(2018 \mathrm{~b}, \S 5)^{10}$ If there is this sort of relationship between intrinsic natures and causal powers it seems at least possible that facts about the latter may provide evidence about the former, and the silence of physics is no longer guaranteed.

By way of illustration let's turn again to the Standard Model. In the case of the quarks the similarities in behaviour between the different generations are sufficiently great (the generations differ principally solely in mass) that there is clearly a strong case for holding that we are dealing with the same kind of entity in all three generations. However, when we turn to consider particles in different families there are significant differences in patterns of behaviour. While the quarks are bound together via the strong interaction, the leptons cannot participate in the strong interaction and bound only by electro-magnetic and weak forces. Leptons can exist on their own, quarks cannot: they're always bound to other quarks. Among the six leptons three are electrically charged (the electron, the muon, the tauon) whereas the three types of neutrino are electrically neutral. Among the bosons, the photon and gluon are entirely massless, and are only able to travel at the speed of light, other particles have mass and travel at less than light-speed - a feature whose implications we will be considering shortly.

One of the most significant differences separates the fermions from the bosons. Whereas no two fermions can share the same quantum state, bosons are not restricted in this way: any number of bosonic particles (of the same type) can share exactly the same quantum state. In effect, fermions behave like classical material objects in that it is impossible for more than one of them to occupy the same region of space at the same time. Indeed, it is because electrons are fermions that matter as we know it is possible: the electrons orbiting an atomic nuclei cannot enter the same quantum state, and so have to occupy different orbits-they cannot all occupy the same low-energy ground state. Photons are bosons, and in everyday life we will all have encountered cases where light beams effortlessly pass through one another without any interference. If ordinary matter were composed of bosons instead of

\footnotetext{
9 Strawson suggests that two physical systems can't be identical in all possible behavioural respects if they possess different intrinsic natures: “... it's indefensible metaphysical separatism, 'stuff/law separatism', to think that there is on the one hand stuff, substance, and on the other hand laws that govern it... The laws are constitutive of the being of the stuff ..." (Strawson 2017c, p. 187). Strawson has consistently defended this view for some time, see his (1987) and (2008a).

10 Also see Mørch (2018a, 2019).
} 
fermions we would be able effortlessly to walk through walls or pass through floors; but since they aren't—for better or worse-we can't.

Suppose Strawson and Mørch are right when they claim that if an object is causally disposed to behave in certain ways in certain circumstances it is because it has a certain kind of categorical nature. If this view is correct, if intrinsic natures determine the causal powers of their possessors, then it is legitimate to take the ways objects are disposed to behave as potential evidence regarding their categorical natures. Given that the causal properties of the elementary particles of the Standard Model diverge in profound ways, to conclude that all types of fundamental particle have an experiential nature because some do looks to be a highly questionable move for the panpsychist to make. If intrinsic natures determine an entity's causal powers the hypothesis that (say) the fermions and bosons have different intrinsic natures looks to be as reasonable as the panpsychist's claim that they all have fundamentally the same type of intrinsic nature.

Panpsychists may well be inclined to respond along the following lines. Radical behavioural differences do exist among the fundamental particles and fields postulated in current physics, but in accepting this we should not lose sight of the fact that when it comes to their modes of behaviour there are also deep commonalities: they exist and persist in space and time, they possess energy and interact causally in accord with physical laws. The panpsychist can maintain that these similarities in very general behavioural tendencies can plausibly be taken as evidence that the entities in question possess the same general kind of intrinsic nature-and this general kind is experiential in character. ${ }^{11}$

A panpsychist reply along these lines certainly has some force. However, it remains the case that if an object's intrinsic nature determines its behaviour, then radical behavioural differences can also reasonably be taken as evidence that differences of the most fundamental kind exist at the level of intrinsic natures. If as Strawson maintains an object's intrinsic nature determines its modes of behaviour it is natural to suppose that intrinsic natures that differ only slightly in character will be associated with broadly similar modes of behaviour, and correspondingly we might expect radically different intrinsic natures to be responsible for modes of behaviour that differ radically. As we have seen, if the picture painted by the Standard Model is correct, some fundamental physical things do behave in radically different ways: some possess mass, others are massless; some are fermions, others are bosons. There are also the forces to consider. There are four fundamental interactions in the Standard Model: the gravitational, the electromagnetic, the strong and the weak. These differ in some quite profound ways. Gravity and electromagnetism extend without limit throughout the entire universe, the strong and weak forces do not extend beyond the confines of the nucleus of an atom. The strengths of the forces also differ markedly, with gravity being far weaker than electromagnetism: when a small toy magnet lifts a paperclip it manages to overcome the gravitational attraction of our entire planet In more precise terms the electromagnetic force is $10^{36}$ times stronger than gravity. The strong force (not surprisingly) is stronger still: it is some

${ }^{11}$ I am grateful to a referee for this point. 
$6 \times 10^{39}$-or six thousand trillion trillion trillion—times stronger than gravity. With behavioural divergences of this magnitude, the suggestion that there are radical differences among the intrinsic natures found in the physical world does not seem far-fetched.

In some of his more recent writings Strawson has suggested that it may be part of the meaning of "physical" that everything physical is made of fundamentally the same kind of stuff, where sameness of stuff-kind equates to possessing fundamentally the same type of intrinsic nature. ${ }^{12}$ Needless to say, if this were the case then panpsychists would be home and dry: from the fact that some parts of the physical world have an experiential intrinsic nature (regions in our brains) we could safely and securely draw the conclusion that everything physical has an experiential inner nature. But the claim in question strikes me as highly questionable. Given the degree to which intrinsic natures are empirically inscrutable, it would be surprising to discover that they played any significant role in our ordinary common sense conception of the physical world. Indeed, it is not difficult to make a plausible case for supposing that when it comes to "being physical" it is solely properties of the causalstructural variety that matter.

Let's suppose that a few thousand years ago some technologically advanced aliens passed through our solar system in their spaceship. By way of a small amusement, these aliens deployed their advanced technology to alter the intrinsic natures of all the matter in the Great Pyramids of ancient Egypt. Previously this matter had the same fundamental intrinsic nature as the rest of the matter in this region of the universe; henceforth it had a very different intrinsic nature. However, since the aliens ensured that the causal-structural properties of the Pyramid-matter remained precisely the same, the switch in intrinsic natures had no effect whatsoever at the level of empirically detectable properties. The pyramids looked the same as they ever did, they smelt and felt as they always did - their constituent rocks passed Dr Johnson's "kick" test effortlessly. When human science developed to the point that chemical analyses of the pyramid matter became possible, it all seemed like perfectly ordinary matter. In a more radical vein we can suppose that last week the aliens returned and-in a more mischievous mood-altered the intrinsic natures of all the Earth's water molecules, but ensured that their causal powers remained exactly the same. Given the latter, none of us noticed that anything untoward had happened. Water looked and tasted the same, food and beverages looked and tasted the same, sailors at sea were oblivious to the change- the fish likewise.

When considering scenarios such as these the suggestion that the altered entities no longer belong to physical reality at all does not seem in the least plausible: it seems just obvious that they do. In this light Strawson's claim that it is part of the meaning of "physical" that everything physical has the same basic stuff-nature looks decidedly implausible. What does seem (highly) plausible is that so far as

\footnotetext{
12 "I also take it to be part of the meaning of the word 'physical' that everything physical has a single fundamental metaphysical nature - a single fundamental stuff-nature that we denote by the word 'physical' ... It follows immediately that physicalism is a monist view, a stuff-monist view, according to which there is only one fundamental kind of stuff in reality." Strawson (2020, p. 326)
} 
membership of the physical realm is concerned, taking up room in physical space and causally interacting with other physical objects and our sensory systems are of paramount importance. $^{13}$

\section{Contingency to the Rescue?}

Not all panpsychist's follow Mørch and Strawson's lead on the relationship between the categorical and the causal. For Goff (2020) the connection between the two is entirely contingent. An object's possessing a given type of intrinsic nature is compatible with its possessing a vast range of different causal powers, and objects that behave in the same ways in the same sorts of circumstance might possess very different categorical natures. If the contingency view is correct, then we cannot take the behavioural differences as evidence for differences in categorical natures. Bosons and fermions may behave very differently, but this is perfectly compatible with their possessing the same type of categorical nature, and so both could be intrinsically phenomenal.

However, it would be a mistake to conclude from this that considerations of ontological economy and simplicity lead straight to panpsychism. A considerably more economical account of the nature of physical reality is available: the view defended by causal structuralists. According to the latter, when we have fully described the causal dispositions of an electron we have exhausted everything there is to say: electrons don't possess intrinsic or categorical natures in addition to their spatio-temporal and dispositional properties. And what goes for electrons goes for all other types of physical entity. Panpsychists accept that the super-austere picture of the physical offered by the causal structuralists has an elegant simplicity, but argue that it is metaphysically incoherent. Goff approvingly quotes Russell's line on this issue: "There are many possible ways of turning some things hitherto regarded as 'real' into mere laws concerning the other things. Obviously there must be a limit to this process, or else all the things in the world will merely be each other's washing."14

Let's suppose Russell and the panpsychists are right, and the standard form of causal structuralism is incoherent. One response- the response adopted by panpsychists - is to hold that all physical entities possess a categorical nature of some kind. A second response is to hold that only some types of physical entity possess a categorical nature-of an experiential kind, we can suppose-whereas others do not.

\footnotetext{
13 There is a more to be said in this connection. In considering just this sort of scenario Strawson (2020, p. 326) suggests that anyone who thinks the altered material must be physical given the undetectable nature of the changes has succumbed to a misguided anti-metaphysical instrumentalism. John Foster has forcefully defended the thesis that the physical is topic-neutral on a number of occasions, and responds to the allegation of verificationism thus: “...there is a special reason why our understanding of physical spatial arrangement has this verificationist aspect. The physical world, to qualify as the physical world ... has to be our world, and it can only be our world in the relevant sense if it is ours empirically-if it is a world that is, as we might put it, empirically immanent." (Foster 2008, p. 138) Also see Foster (1982, Part II).

14 See Goff (2017b, §3.2) and Russell (1927, p. 325).
} 
For the latter kind of entity the account provided by the causal structuralist is correct. Since this hybrid view ascribes categorical natures to fewer physical entities than the panpsychist, it is arguably more economical. Moreover, the hybrid view is immune to the objections panpsychists (and others) deploy against the orthodox structuralists. Since on the hybrid conception some parts of the physical world do possess intrinsic natures - up quarks, down quarks and electrons, say-it is no longer the case that everything is making a living by taking in the washing of other things. Particles that lack intrinsic natures gain ontological respectability by virtue of being able to causally interact (directly or indirectly) with particles which do possess intrinsic natures, whether experiential or non-experiential. Perhaps on further scrutiny this hybrid view will also turn out to be metaphysically problematic, but if so it is something that panpsychists need to demonstrate. ${ }^{15}$

This point aside, it should also be noted that Goff's contingency version of panpsychism comes with at least one distinct disadvantage. For many of its proponents if not all, the constitutive form of panpsychism is an attractive doctrine because placing consciousness at the very heart of matter secures for experiential properties a place in the causal scheme of the physical world that competing approaches - such as property dualism-cannot rival. But as critics have been quick to point out, if phenomenal properties are only contingently connected to their causal roles, the risk of epiphenomenalism looms large. ${ }^{16}$ To illustrate, let's suppose that in our world electrons all have the same sort of intrinsic nature: a particular shade of phenomenal yellow. If the contingency view is correct, a possible world exists which is indistinguishable from ours in all respects save one: the particles which occupy the role of electrons in that worlds physics and chemistry have a particular shade of phenomenal green as their intrinsic nature. Now consider another possible world, one where half of the electron-counterparts have yellow as an intrinsic nature, and half have green.

In making sense of these scenarios it is natural to distinguish two sorts of property: one the one hand there are the intrinsic or categorical natures, and on the other there are the causal-behavioural properties-we can call these the I-properties and the $C B$-properties respectively. In the possible worlds just introduced all the electron-counterparts possess exactly the same CB-properties, but the I-properties are distributed differently. With this distinction drawn we can consider a fourth and final scenario. In this world all the electron-counterparts possess the same intrinsic nature as in the actual world—phenomenal yellow, we are supposing — but they possess different CB-properties: the repulsive force they exert in the proximity of other negatively charged particles is double what it is in the actual world.

When considering worlds such as these a question arises concerning the electroncounterparts: are they really electrons, or particles of a different type? We needn't

\footnotetext{
15 The kind of universe-wide fields that are prominent in current physics are helpfully relevant here. If one of these spatially extended fields were to possess an experiential intrinsic nature, it could provide particles construed as mobile clusters of causal powers with a location in physical space-something which otherwise prove problematic.

16 For example see Howell (2015) and Brüntrup (2016).
} 
try to answer that question here. For present purposes all that matters is that it seems plausible to hold that for each of these worlds, when it comes to explaining how and why these particles act as they do it looks as though it is only the CB-properties which matter. In scenarios where the intrinsic natures vary but the CB-properties are unchanged, the particles' causal powers are remain the same; if the CB-properties change, so too do the causal powers-irrespective of whether the intrinsic natures remain the same or vary. The I-properties are causally irrelevant. Intrinsic natures may be located within the physical world, but they are contributing nothing to our understanding of how and why the world works as it does. In this respect the panpsychist's position seems no better than epiphenomenalist variants of property dualism, where phenomenal properties exist, but cannot causally intervene in the physical world.

This result can easily be averted: the panpsychist can follow Strawson and Mørch in holding that intrinsic natures determine causal behaviour. But as we have also seen, if they make this move the silence of physics is undermined: the ways in which physical things are disposed to behave serves as evidence for the kind of intrinsic natures they possess - and in the case of the Standard Model the very different behaviours of bosons and fermions, or the dramatic differences between the strong and gravitational forces, can be taken as evidence that some constituents of the physical world possess fundamentally different types of intrinsic nature.

Before moving on there is a further point worth registering. We have just seen that the plausibility of the panpsychist's case depends in part at least on how representative the kind of physical stuff in our brains is with respect to the rest of the physical world. Thanks to developments in cosmology this question is currently harder to answer than it has been for centuries.

In recent decades the problems of dark matter and dark energy have gradually grown in prominence. As has been widely reported, it is now believed the kind of matter found in planets and stars - and our brains — only makes up around 5\% of the universe, with around $68 \%$ being composed of dark energy and the remaining $27 \%$ being made up of dark matter. All that is known about dark energy is that it responsible for the universe's accelerating rate of expansion. All that's known about dark matter is that it exerts a significant gravitational influence on the stars inside galaxies but does not interact with ordinary matter in any other known way-hence the difficulty of detecting it.

Since none of the particles in the Standard Model has these characteristics, dark matter must be composed of a hitherto unrecognized kind of particle or field. Physicists have conjured up a large number of dark matter candidates: WIMPs, GIMPs, sterile neutrinos, axions, super-symmetric neutralinos, dark photons, heavy photons, condensed scalar fields - the latter consisting of a galaxy-sized Bose-Einstein condensate which is (in effect) a single particle. Experimental evidence for the existence of any of these candidates has thus far proved elusive, but the search goes on. ${ }^{17}$

Given that these postulated inhabitants of the "dark sector" of the physical world differ from the known kinds of matter in very profound ways it may well turn out

17 See Feng (2010) for a review of some competing theories concerning dark matter. 
that the matter in our brains is far less representative of the matter in the wider universe than we have hitherto been assuming. We will have to wait for future discoveries in astro-physics to learn just how unrepresentative we are.

\section{Energy and Material Interconvertibility}

When a caterpillar turns into a chrysalis which in due course becomes a butterfly the process of metamorphosis is such that Nagel's reasoning looks very plausible. Since the same elementary particles which come to constitute the butterfly previously constituted (some of) the interior of the chrysalis they are bound to possess the same intrinsic nature-an experiential nature, if the panpsychists are right. Although at the level of elementary particles the Standard Model permits a wide range of transformations, the nature of these transformations is quite different from biological metamorphosis, and there is no obvious guarantee at all that they preserve intrinsic natures. To illustrate, suppose an electron collides with a positron to produce two photons. Since electrons, positrons and photons are all elementary they do not possess constituent parts, and so there is no possibility of the electron (say) passing on some of its parts to the resulting photons-the electron and positron are completely annihilated when they collide, and the photons that are created out of the collision are completely new particles. Although it's possible that the photons possess an intrinsic nature of the same fundamental kind as the positron and electron, it's also possible that they do not. The situation is quite unlike the caterpillar-butterfly case.

In a number of recent writings Strawson has put forward a line of argument that, were it successful, would establish that all types of physical entity are interconvertible in the way Nagel's case for panpsychism requires. The reasoning can be summarized thus ${ }^{18}$ :

(1) Everything physical is constituted of energy: matter is energy,

(2) Different forms of matter are simply energy in different forms.

(3) All forms of energy possess the same fundamental intrinsic nature.

(4) All forms of energy are interconvertible.

(5) Given (2) and (4) any form matter is convertible into any other form (2017a, p. 14).

(6) If the matter and energy in our brains have an experiential intrinsic nature then all matter and energy have an experiential intrinsic nature, given (1) and (3)

(7) There are material processes in our brains which possess an intrinsic experiential nature.

(8) So everything physical has an experiential intrinsic nature-given 6 and 7.

In support of (1) Strawson draws on the authority of Heisenberg, who claimed that "All the elementary particles are made of the same substance, which we may call

\footnotetext{
${ }^{18}$ Here I am reconstructing a line of argument found in a number of Strawson's writings-for a recent example see his (2017a; 2017b).
} 
energy or universal matter ..." and who took the constitution of matter by energy to be "the final proof of the unity of matter" $(1958,160) .{ }^{19}$

When endorsing the Heisenberg position Strawson tells us that he "is putting aside the common use according to which 'energy' denotes the power of 'doing work' contained in or possessed by a body or system of bodies." This is a helpful clarification, but also a little misleading. As Strawson is clearly well aware, the "common use" he is setting aside is not just the view of the educated person in the street (if there is such a thing in the case of the nature of energy). He is also overlooking the current orthodoxy in contemporary physics, where energy is generally not conceived as a homogeneous substance of any sort. ${ }^{20}$

If the concept of energy plays a central role in physics today it was not always the case. It started to take center-stage only during the nineteenth century, when physicists such as Mayer, Joule and Helmholtz came to recognize that very different physical properties could be related in precise quantitative ways. Joule, for example, demonstrated in the 1840's how to connect the amount of work needed to lift a given mass to a certain height to the amount of heat required to raise the temperature of a certain quantity of water by a certain amount. Joule also found accurate ways of quantifying electrical current and voltage, and went on to conduct experiments which revealed the relationship between the strength of the current and voltage flowing into an electric motor with the weight it is capable of lifting and extent to which the motor heats up.

Rather than an ingredient of reality in its own right, energy in this guise is akin to a collection of book-keeping rules which allow us to relate quantitatively a (very) diverse range of physical properties which are ingredients of reality in their own right. Here is Feynman characterizing the law of the conservation of energy in his Lectures on Physics:

It states that there is a certain quantity, which we call energy, that does not change in the manifold changes which nature undergoes. That is a most abstract idea, because it is a mathematical principle; it says that there is a numerical quantity which does not change when something happens. It is not a description of a mechanism, or anything concrete; it is just a strange fact that we can calculate some number and when we finish watching nature go through her tricks and calculate the number again, it is the same. $(1964, \S 4.1)$

Feynman here is clearly directing us away from thinking of energy as any kind of concrete entity in its own right. ${ }^{21}$

The fact that "E" figures in so many physics equations makes it natural to think that there must be some single physical entity to which it corresponds, but there need be no such entity for the equations to function as they do. ${ }^{22}$ In this connection

\footnotetext{
19 Also: "energy is a substance, ... all particles are made of the same stuff: energy" (1958, p. 63, 71).

${ }^{20}$ Hence Strawson remark "I take this general position to be orthodoxy today (no doubt this is unwise)." (2017a, §1).

21 For a useful overview of energy-related misconceptions see Strassler (2012).

${ }^{22}$ It is not for nothing that energy has been described as the "subtle" concept—see Coopersmith (2015).
} 
it's true that physicists themselves sometimes talk in misleading ways when communicating with the wider public-Heisenberg is far from alone in this regard. By way of illustration: on CERN's webpage concerning the matter-antimatter asymmetry one can read "Matter and antimatter particles are always produced as a pair and, if they come into contact, annihilate one another, leaving behind pure energy". This is quite misleading. If an electron and a positron meet and annihilate one another, what's produced are other particles, e.g. a pair of photons, or (if they're moving fast enough) a muon and anti-muon. These annihilation-products certainly have energy, but they're not just energy.

In a similar but also potentially misleading vein, physicists do often talk of "energy being released" in a nuclear explosion thanks to Einstein's equation $E=m c^{2}$. Again, what is created by an atomic explosion is not "pure energy" but a range of much more specific effects. When a nuclear bomb is triggered the ensuing nuclear reaction generates X-rays, which induce an enormous and rapid increase in temperature (i.e. more rapid molecular motions) in the atmosphere in the immediate vicinity of the bomb; this rapid temperature increase-millions of degrees centigrade - produces the blast wave which devastates the surroundings. The explosion also large quantities of light, radiant heat and gamma radiation (i.e. electromagnetic waves of differing wavelengths) as well as radioactive particles. "Pure energy" features nowhere in this picture, which is complete without it.

There is a further reason why taking energy to be a basic physical ingredient in its own right is problematic. In post-relativity physics it is orthodoxy to regard the basic physical properties as those that are relativistically invariant, i.e. those properties which do not vary from one inertial reference frame to another. If a material object is in motion one part of its total energy will derive from its kinetic energy, where $\mathrm{KE}=\mathrm{mv}^{2}$. Since the velocity of this object will vary from one reference frame to another, so too will its kinetic energy. The energy of material bodies is thus not a relativistic invariant in special relativity. ${ }^{23}$

There is a great deal more to be said about these matters, but it doesn't need saying here. Since Heisenberg's conception of energy as "universal matter" is at least as controversial as the doctrine of panpsychism - at least as things stand in contemporary science-invoking it in support of panpsychism adds nothing substantial to the case for the latter.

\section{Being Light}

Time to return to some unfinished business. Earlier when discussing the various differences between the Standard Model's fundamental particles I pointed out that whereas some types of particle possess mass, other types are entirely massless. Since this difference may prove particularly troublesome for the panpsychist it merits a separate treatment.

\footnotetext{
${ }^{23}$ For more on this see Lange (2001) and (2002, chapter 8).
} 
In his Autobiographical Notes Einstein relates a striking thought experiment he conducted during his teen years:

...a paradox upon which I had already hit at the age of sixteen: If I pursue a beam of light with the velocity c (velocity of light in a vacuum), I should observe such a beam of light as an electromagnetic field at rest though spatially oscillating. There seems to be no such thing, however, neither on the basis of experience nor according to Maxwell's equations. From the very beginning it appeared to me intuitively clear that, judged from the standpoint of such an observer, everything would have to happen according to the same laws as for an observer who, relative to the earth, was at rest. For how should the first observer know or be able to determine, that he is in a state of fast uniform motion? One sees in this paradox the germ of the special relativity theory is already contained. (Einstein, 1949)

Exactly what lesson Einstein drew from this famous thought experiment has been debated-it's not altogether obvious. But I want to focus here on a related thought experiment: what would it like to be a beam of light?

For Einstein, the answer would probably be "nothing", since-as far as I'm aware-he wasn't a panpsychist. Let's suppose, however, that the panpsychists are right when they maintain that each and every constituent of physical reality has an experiential intrinsic character of some kind. Since light rays are clearly parts of physical reality, they too must have some sort of experiential intrinsic character. Consequently, there must be something that it's like to be a light-beam. Or to put it another way, photons must be conscious beings. However, a generally accepted consequence of Einstein's special theory of relativity (STR) makes envisaging what it's like to be one of these conscious beings problematic.

Let's suppose you are in a super-powerful spaceship with a large clock firmly glued to its side, travelling ever faster in pursuit of a stream of photons. As your speed steadily increases, from the inside of the spaceship you'd notice nothing unusual. The objects you can see around you would look and behave in perfectly normal way. When playing a game of catch with a crew-mate the ball would travel back and forth through the air in a normal way; eggs would take four or so minutes to boil; your crew-mates would seem to walk and talk at the normal rate-your own thoughts and sensations would seem to flow at the normal rate. But for people on the Earth, say, watching you through a telescope, your ship would appear to be contracting length-wise, and the external clock ticking ever more slowly. Thanks to your increasing speed, your "proper time" - the rate at which physical processes occur in your inertial frame of reference-is becoming increasingly slowed with respect to the proper time of the Earth. When you reach $99.5 \%$ light-speed observers on Earth would see your clock ticking just once every ten seconds. As you ever closer to light speed the clock would be seen to tick ever more slowly, and the length-contraction becomes extreme.

If you were to actually achieve light-speed your spaceship's clock would be seen by Earth-side observers to stop altogether-and for your spaceship and everything aboard it the passage of time would have come to a complete halt. Due to relativistic mass-gain as speeds increase, a material object such as a spaceship can ever attain 
the speed of light, but light obviously can and does. For a ray of light time has effectively stopped. For those of us travelling at less than light speed this is not obvious. A photon emitted by the sun will be measured as taking nine minutes to reach the Earth, a photon emitted from a distant region of the Milky Way can take thousands of years to reach us. But from the perspective of the photon itself the situation is very different: for a photon such journeys take no time at all. Between their departure and arrival the proper time elapsed is zero, irrespective of the spatial distance they have travelled.

Viewing matters from the vantage point of Minkowski spacetime-as contemporary physics generally does-provides a useful further perspective. The speed of light $c$ is an invariant in Minkowski spacetime: it has the same value for observers, irrespective of how fast they are moving relative to one another or in what direction. Another invariant is the so-called spacetime interval between events, whose magnitude is determined by this formula:

$$
[\text { spacetime interval }]^{2}=[\text { separation in time }]^{2}-[\text { separation in space }]^{2}
$$

Although observers who are moving with respect to one another will typically disagree about the spatial and temporal distances separating a given pair of events, all observers will invariably agree on the magnitude of the spacetime interval separating these two events. The paths of light rays lie on "null geodesics" in Minkowski spacetime, and the spacetime interval separating any two locations along a null geodesic is another invariant: it always has the value of zero, with the relevant points having zero spatial separation and zero temporal separation. A light ray travelling through empty space takes no time to reach its destination because from its perspective the distance it travels has shrunk to nothing. ${ }^{24}$

Why does any of this make for a problem for there being something it's like to be a ray of light? If you pause for a moment and try to imagine precisely what it would like to be something for which time did not pass you will very likely find it very difficult. This is simply because our own waking consciousness is typically continuously changing in some way, and the attempt at imagining a timeless experience is itself a component of our stream of consciousness. Even if we manage to bring our inner soliloquy to a halt (a notoriously difficult achievement in itself), there will remain a constant background flow of sensory experience-and if we try to bring this to a half we simply fail. For these reasons most phenomenologicallyinclined philosophers have held that consciousness is essentially temporal in nature. Any form of consciousness, even the simplest contains some experienced change or

\footnotetext{
${ }^{24}$ In diagrams depicting Minkowski spacetime light-like trajectories are drawn as lines which have a certain spatial length, but as Wilson points out, these need to be interpreted in the right way: "The situation can be compared to a map of the Earth's surface in a flat projection (though the analogy is of course not exact). The North and South poles each correspond to an entire edge of such a map, by convention the top and bottom edges respectively. Any route along the top or bottom edge of a map like this has zero length, even though it looks extended on the map. The map is misleading concerning lengths across the Earth's surface in the limiting case of the North and South poles, just as a Minkowski diagram is misleading concerning proper times elapsed in the limiting case of photon trajectories." (Wilson, 2018, §7) In this connection also see Rickles (2016, p. 71).
} 
experienced flow. James, Bradley, Bergson and Husserl all subscribed to this viewas does Strawson (2008b, §8.6).

Let's call the essential temporality of consciousness doctrine ETC, which we can take to include the following combination of claims:

ETC: all forms of consciousness, from the most complex to the simplest possible, are essentially temporal: all possess experienced flow. Experienced duration necessarily possesses some temporal depth, and as such cannot exist in a durationless instant.

If ETC is true then there's nothing it's like to be a photon, or a beam of light, or any other massless entity travelling at light speed-such as a gluon or gravitational wave. As entities that are timeless and changeless, they cannot possess an inner experiential nature characterized by phenomenal flow or change if the latter requires a temporally extended interval in which to exist or unfold. In a recent discussion Roger Penrose makes this observation:

$\ldots$ it is pretty hard to bore a photon or a graviton-even aside from the unlikelihood that such entities have significant experiences! The point is that, according to a photon or graviton, the passage of passage of time is as nothing. Such a particle can even reach eternity before encountering the first "tick" of its internal clock ... One might well say that "eternity is no big deal" for a photon or graviton! (Cycles of Time, 2010, 146)

If panpsychism is true photons must have experiences of some sort, even if feeling bored is beyond them. But there is a deeper problem here that Penrose seems to have missed. If photons are timeless and consciousness is essentially temporal, it seems photons could be unable to have any sort of experience at all.

Returning to the macro-level for a moment, I think most panpsychists would agree that if a human being were to step into a temporal stasis machine that brings all physical processes at all scales of magnitude to an absolute stop for a period of ten minutes, then the consciousness of that person would also come to a stop, and not continue on through the relevant period. Why should it be any different for photons and beams of light? If they are temporally frozen they too can't be conscious.

As noted earlier, from the vantage point of relativistic quantum field theory-currently the best physics has to offer-photons are not viewed as autonomous classical particles, but as discrete units of mobile excitation in a constantly oscillating quantum electromagnetic field that fills the entirety of spacetime. As with all things quantum, light can seem to behave in a wave-like or particle-like manner, depending on the circumstances:

According to quantum field theory, when we observe a field carefully enough we see it resolve into individual particles_although the field itself is real. (The field actually has a wave function describing the probability of finding it with any particular value at each point in space.) Think of a TV set or computer monitor, which seems to display a smooth picture from a distance, but close up we find that it's actually a collection of tiny pixels. On a quantum TV set there really is a smooth picture, but when we look closely at it we can only ever 
observe it as pixels. ... So light is a wave, a set of propagating ripples in the electromagnetic field that pervades space. When we throw quantum mechanics into the mix, we end up with quantum field theory, which says that when we look closely at an electromagnetic field we see it as individual photons. (Carroll, 2012, pp. 129-30) ${ }^{25}$

Strawson has a fondness for this sort of field-conception since it makes it easier (for him at least) to envisage how physical things could be conscious. ${ }^{26}$ Does moving from a classical to a quantum theory of light make a difference to the situation? In one sense it makes no difference at all: precisely by virtue of being relativistic the Standard Model's field theories are all fully compatible with Special Relativity, and so electromagnetic processes remains massless and timeless. But in another respect the situation becomes more complex, simply because there is no agreement on how quantum theory or quantum field theories are best interpreted, and the different interpretations provide very different pictures of physical reality.

As Strawson notes, one possibility is that the particle-like excitations in a quantum field are analogous to waves of water moving across the surface of an ocean. ${ }^{27}$ If this were the case then fields and particles would (in effect) be made of the same kind of stuff-a wave of water is nothing but water-in-motion - and it would be very difficult to see how photons could have a fundamentally different nature than their field. In which case, since being temporal or atemporal is a difference in fundamental nature, if photons are timeless then the entire electro-magnetic field will be as well. There may be alternative ways of construing physical fields, but it is not clear that any are open to a panpsychist.

As for why, let's assume-with quantum field theorists—-that quantum fields are physical entities of a fundamental sort. If panpsychism is true these fundamental entities will have an experiential intrinsic nature. The only obvious form of spacefilling experience that seems fit for purpose is a sense-field. A two- or three-dimensional expanse of (phenomenal) pale blue extends through space in the same general sort of way as a quantum field. All physical fields-whether quantum or classical-involve an assignment of a collection of properties to every point in spacetime. Since sense-fields are plenums they are perfectly equipped to play this role. If we now envisage small regions of dark blue moving through our pale blue phenomenal expanse then we have an approximate experiential analogue of the mobile particlelike regions of excitation in a quantum field.

\footnotetext{
${ }^{25}$ For a very useful attempt to capture some of the essentials of the theory pictorially see Skinner (2015).

${ }^{26}$ I have in mind passages such as this one: “... lumpish, inert matter, dense or corpuscled, stuff that seems essentially alien to the phenomenon of consciousness, has given way to fields of energy, essentially active diaphanous process-stuff that—intuitively—seems far less unlike the process of consciousness. ... For what, expressed in common-sense terms, does physics find in the volume of spacetime occupied by a brain? Not a sludgy mass, but an astonishingly (to us) insubstantial-seeming play of energy, an ethereally radiant vibrancy" Strawson $(2003, \S 14)$.

${ }^{27}$ See Strawson (2006b, p. 228). From an idealist standpoint Kastrup suggests (2019, p. 137) that we seem to inhabit the same world because we are "surrounded by the same universal field of phenomenality like whirlpools in a single stream."
} 
In an experiential space of this kind the field-contents and the field itself are of the same general experiential type: they possess natures of a single determinable kind. ${ }^{28}$ If the relationship between quantum fields and their contents is analogous to this, contents and field too must have the same general intrinsic nature-a nature of an experiential kind if the panpsychists are right. Hence in the case of the electro-magnetic field, if the regions of excitation are timeless, the entire field will be. Perhaps there are alternative ways of making sense of the relationship between fields and field-contents in a wholly experiential way that are equally plausible. If so panpsychists might want to tell us what they are.

\section{A Path to Darkness}

In quantum field theories the graviton carries the gravitational force, and consists of a massless light-speed vibration in the gravitational field which determines the structure of space-time. If the gravitational field is timeless, and timeless experience is impossible, it may well be that yet another portion of reality will be excluded from the realm of the experiential. But since the graviton remains hypothetical-a satisfactory theory of quantum gravity proving elusive- the panpsychist may feel justified in not losing too much sleep on this score, at least for now. ${ }^{29}$ The situation with regard to the electromagnetic field is very different: its timeless character is a direct consequence of Einstein's special relativity, a well-established part of physics that those seeking to develop quantum theories of gravity are keen to accommodate.

There are a number of potential moves open to the panpsychist when confronted with the problem posed by the timeless nature of light. One option is to hold that experiences with temporally extended phenomenal contents need not themselves be temporally extended in ordinary objective time. There are those-e.g. Brentano, Husserl, the early Broad-who advocate this position for reasons that are quite independent of relativistic physics. If phenomenal contents that are seemingly temporally extended can exist within conscious episodes that are strictly durationless it is no long absurd to suppose that a photon might have such an experience. ${ }^{30}$ However,

\footnotetext{
${ }^{28}$ I am not suggesting the sense-field must be visual, there might be many different forms of experience that can be field-like. Remaining in the realm of human experience, the surface of our skin might serve - think of what it is like to feel a fly crawl across one's skin. For more detailed discussions of the properties of sensory fields and their ability to replicate the properties of physical space see Foster (1982, chapter 7) and Dainton (2014).

29 The same applies to those speculative approaches to quantum gravity according to which space and time are emergent phenomenon, and the underlying reality is wholly timeless - see Schneider (2018). The so-called "quantum zeno effect"-see Patil et al. (2015)—also produces temporally frozen physical systems. The existence of such states poses a more immediate problem for the panpsychist, though if as seems likely such states are extremely rare the problem is comparatively small in scale. If, as some have speculated, the matter inside black holes is temporally frozen the scale of the problem confronting panpsychists would be significantly greater-but we will have to wait for advances in black hole physics before we can be confident of making claims about their interiors. We will be taking a look at the gluon field later on.

30 See Dainton (2017) for more on these competing approaches to the temporality of consciousness.
} 
this won't be an option for those who-like Strawson-are hostile to the idea that anything concretely real can be strictly dimensionless spatially or temporally. ${ }^{31} \mathrm{~A}$ more promising option might be to turn to physics itself for support. The doctrine that light is a wavelike entity in its own right that pervades space is firmly established in contemporary physics - as a brief browse through any textbook will confirm-but there are alternative conceptions.

When Maxwell's field-based theory of electromagnetism emerged in the 1860's its main rivals were direct action (or "action-at-a-distance") theories along the line of Newton's highly successful account of gravity. According to the latter, every object in the universe is continually exerting an attractive force on every other object in the universe, a force that weakens with distance. Accordingly, if you lift up your arm, everything in the universe is instantaneously affected, even if only by a tiny amount-e.g. since your arm is now closer to distant stars and galaxies directly above you, the gravitational attraction between you and the stars increases slightly. For the pre-Maxwell direct action theorists, in the electrical case a similar attractive (or repulsive) force exists between charged bodies, a force which weakens with distance. Like Newton's gravity, this hypothetical electrical force acts instantly and directly between bodies, irrespective of the distance separating them. In sharp contrast, from the vantage point of Maxwell's theory such effects are all dependent on a space-pervading electro-magnetic field, with the consequence that the relevant forces are transmitted from one region of space to an adjacent region of space- they do not affect distant bodies directly or instantaneously.

Although the field-based theory of electromagnetism and other basic interactions) remains dominant in contemporary physics, direct action alternatives have been developed. Probably the best-known derives from Feynman's early work, and is often referred to as the "Feynman-Wheeler" (F-W) theory, from the title of a paper jointly authored with his doctoral supervisor J.A. Wheeler. ${ }^{32}$

We naturally think of electromagnetic processes as being temporally asymmetrical. Suppose you switch on a radio transmitter and send a pulse of radio waves to nearby star, and emissions from the transmitter travel through space towards the star and reach it after five years. In such cases we tend to assume that the only causally relevant factor are the emissions travelling from the Earth - the distant star has no inkling (as it were) that a radio signal is heading in its direction from Earth until the signal arrives. If the F-W view is correct the real state of affairs is quite different. In addition to the "retarded" signal emitted from the Earth to the star, there exists an "advanced" signal emitted back through time from the star to the Earth. These two influences largely cancel each other out leaving only the measurable result: a transfer of energy between the Earth and the star, a transfer which consists of a direct causal interaction between the two bodies-a connection which does not require any intervening medium or field.

Feynman was led to consider time-delayed direct action alternatives to Maxwell's theory in an attempt to solve some intractable problems that afflicted

\footnotetext{
31 Strawson has confirmed as much in correspondence.

32 See Wheeler and Feynman $(1945,1949)$.
} 
electromagnetic theory in the 1930's. A particle that is electrically charged (e.g. a proton) requires more force to accelerate it to a given speed-it has greater inertia-than a particle that lacks a charge (e.g. a neutron), even when the particles have similar mass. Explaining this "radiation resistance" (as it was known) in terms of interactions between charged particles and the surrounding electromagnetic field was the natural way to go, but developing a viable account along these lines had proved difficult. A second problem was the so-called "self-energy" of the electron. This quantity was a product of the electron's rest mass, and its interactions with the electromagnetic field. If an electron is a point-sized entity (as was assumed to be the case) calculations showed that the electron's field-interactions would lead to its possessing an infinite self-energy. Feynman reasoned that if the problematic infinite energy derived from the electron's interactions with the electromagnetic field, the problem might well go away if we got rid of the field altogether. As for Wheeler, prior to encountering Feynman the growing evidence that quantum theory is fundamentally non-local had led him to suspect that a reconsideration of direct action theories was long overdue. Having recently read a 1938 paper by Dirac he was also well aware that Maxwell's equations are time symmetric, a point that is often overlooked in expositions of them. If the solutions to Maxwell's equations found in textbooks almost invariably involved waves travelling forward in time, the equations themselves always featured an "advanced" solution, involving waves moving backwards in time. Physicists had long become accustomed to simply ignoring the advanced solutions as a "non-physical peculiarity"-Wheeler thought it well worthwhile exploring whether this stance was misguided.

In their work on what came to be called the "absorber theory" Feynman and Wheeler succeeded in explaining radiation resistance without recourse to an electromagnetic field. The additional inertia charged particles possess is due to sum total of their direct causal interactions with all the other particles in the universe- the need for a field is entirely eliminated. When a charged particle begins to move it emits a signal to all the other particles in the universe. To accommodate relativity these signals do not arrive at their eventual absorbers instantaneously but only after a delay. The magnitude of this delay is exactly equivalent to the time it would take a signal travelling at light-speed through space to reach a distant source-though in this case since the causal influence is direct nothing actually travels through space. When these time-delayed signals reach distant particles, they emit a signal in response in the direction of the emitting particle. By virtue of travelling backwards in time these signals arrive at the precise instant at which the earlier particle emitted its forwardtravelling signal. The increased inertia this charged particle possesses the moment a force impinges upon it is a product of its interactions with particles everywhere else in the universe. The mathematical techniques Feynman developed to deal with this potentially vast number of interactions, demonstrating that they led to a finite quantifiable physical outcome, would be later adapted to serve in his influential "sum over histories" method in quantum mechanics. Wheeler would later summarize the merits of the theory thus:

[the W-F approach] swept the electromagnetic field from between the charged particles and replaced it with "half-retarded, half advanced direct interaction" 
between particle and particle. It was the high point of this work to show that the standard and well-tested force of reaction of radiation on an accelerated charge is accounted for as the sum of the direct actions on that charge by all the charges of any distant complete absorber. Such a formulation enforces global physical laws, and results in a quantitatively correct description of radiative phenomena, without assigning stress-energy to the electromagnetic field ... There are no entities other than the particles; the field itself is removed from consideration, either as a "automatic conserver of the source" or as the conveyor of stress-energy from the accelerating charge. (Wesley and Wheeler 1972, p. $427-8)^{33}$

Despite further work in the 1940's and early 1950's Feynman and Wheeler were unable to solve the self-energy problem, and Feynman turned his attention to other issues.

Feynman and Wheeler may have abandoned their radical conception of electromagnetic interactions but other physicists continued to see the appeal of direct action theories. Fully relativistic and quantum-compatible versions of the absorber theory were developed by Davies $(1971,1972)$, and the approach also found favour for cosmological reasons with the steady state theorists Hoyle and Narlikar (1995). In several papers published in the 1980's Cramer developed the absorber theory several stages further. ${ }^{34}$ Cramer pointed out that just Maxwell's equations for electromagnetism have an often overlooked advanced solution, so too does the fully relativistic version of Schrödinger's equation-it too is fully time-symmetrical and so compatible with influences travelling from the future to the past. Moreover, and more importantly, by allowing advanced solutions to play a role in the evolution of quantum systems many of the problematic aspects of quantum theory simply disappear. The otherwise baffling behavior produced by the notorious "experiment with two holes" is no longer baffling at all-likewise quantum entanglement. ${ }^{35}$ No less importantly, there is no longer any need for a non-quantum mechanical "observer" to produce a collapse in the quantum wave function. When (say) a first atom emits a photon which is absorbed by a second atom, the entire process is a consequence of the interplay (or "transactions") between the advanced and retarded quantum waves emitted by the first atom and all the other atoms with which it is interacting. More recently the merits of the transactional approach to quantum theory have also been championed by Ruth Kastner. ${ }^{36}$ As well showing how the transactional approach can evade problematic counterexamples, Kastner has argued that it provides an advantageous direct action alternative to more orthodox quantum field theories.

Stepping back, it is clear that these developments of the F-W approach are highly relevant to the problem for panpsychism we are considering. It is difficult to see

\footnotetext{
33 For a more detailed account of this work and the background from which it emerged, see Wheeler (1998, pp. 162-167).

34 For an overview see Cramer (1986, 2016).

35 See Gribbin (1995) for a highly readable account of these advantages of the transactional interpretation.

36 Kastner (2012a, 2012b, 2016).
} 
how photons could have an experiential intrinsic nature if they are also intrinsically timeless. But there is only a problem here if photons exist as material entities in their own right. The availability of a direct action alternative to the standard accounts of electromagnetism means that panpsychists have the option of simply denying that photons (as standardly construed) exist. And what goes for photons also goes for the entire electromagnetic field: it too simply does not exist. If panpsychists opt to take this step so as to avoid a serious problem for their position they can do so in the knowledge that are following in the footsteps of a respected (if minority) movement in contemporary physics. ${ }^{37} \mathrm{~A}$ universe without light-as-usually-conceived is in some respects a very dark place - there is certainly less of the luminous radiance that Strawson finds so appealing-but panpsychists may think this is a price worth paying. ${ }^{38}$

\section{Mass Concerns}

Irrespective of whether the panpsychist opts to eliminate light or to retain it in its field-theoretic form, the problem it poses might not seem overly serious. It's true that the electromagnetic field is pervasive, extending as it does through every region of ordinary space-time; it's also true that visible light only constitutes a small portion of the totality of electro-magnetic phenomena-there are also radio waves, $\mathrm{X}$-rays, infra-red and so forth. Even so, the panpsychist could maintain, with some prima facie plausibility, that electromagnetic phenomena don't make up much of the material substance of the universe, the vast bulk of this resides in the vast quantities of matter found in stars and galaxies. If all this matter possesses an entirely experiential intrinsic nature we have something not very distant from full panpsychism-a point which is only reinforced by the discovery that light and other electro-magnetic phenomena are entirely massless.

However, in fact the situation is not so straightforward. As we saw earlier, in contemporary physics the photon is not the only massless particle condemned to travel always at light-speed: gravitons and gluons also fall into the massless/timeless category. Since the graviton remains hypothetical I will not devote any further attention to it here. The situation with the gluon is quite different: it is a firmly established component of the Standard Model. Gluons may be massless but if our current theories are along the right lines, they contribute more to the world's "substance" than one might expect.

In experiments carried out in Manchester in 1909 Rutherford discovered that positively charged alpha particles fired at pieces of very thin gold foil (around 20

\footnotetext{
$\overline{37}$ In this connection see Silberstein et al (2018), a manifesto for developing direct action alternatives in all areas of fundamental physics as a means of avoiding the well-publicized pitfalls of mainstream approaches to quantum field theory, quantum mechanics and general relativity-intriguingly in the present context, they also advocate a form of panpsychism.

${ }^{38}$ Of course panpsychists should be wary of taking this sort of step too often. After all, if they are obliged to eliminate too much of the world-as-we-are-accustomed-to-conceiving-it, the doctrine would soon diminish in appeal.
} 
atoms thick) were sometimes bounced right back. This came as a big surprise. The dominant conception of the structure of atoms at the time was the "plumb pudding" model, according to which an atom is a small positively charged portion of mass, with even smaller negatively charged plumb-like entities distributed evenly through it. If this model were correct, you wouldn't get alpha particles bouncing back in the way they did, they would simply be slowed down as they passed through the positively charged regions of an atom. ${ }^{39}$ The rebound seemed to suggest that positive charge must be far more concentrated. It was not long before Rutherford proposed the new model of the atom which would make him famous. On the new view all the positive charge is concentrated in a tiny core that is at most one thousandth the size of an atom: the proton had been discovered.

Half a century later history was to repeat itself-partially at least. Physicists began to take the existence of quarks and gluons seriously when evidence began to emerge from particle physics experiments carried out in the 1960's and 70's. Beams of high energy electrons fired at protons scattered in ways which suggest that protons must themselves have a complex internal structure. Closer studies revealed that the scattering centres had spin 1/2, as predicted by Gell-Mann and Zweig's quark theory, proposed in 1964. Although the latter did a wonderful job of taming the "particle zoo" it had hitherto lacked firm experimental confirmation. Further work on the quark model led to quantum chromodynamics (QCD) in 1973, a field which is now part of the Standard Model.

In some respects QCD resembles the earlier quantum electro-dynamics (QED): both are relativistic quantum field theories, both posit force-transmitting particles: photons in the case of QED, gluons in the case of QCD. The gluon force which binds quarks is very strong: it is so strong that quarks cannot escape their confinement within protons and neutrons (a "free quark" has never been detected). But the gluon force differs in another way: gravitational and electromagnetic forces get weaker with distance, the gluon force gets stronger with distance. This difference aside, QCD is a good deal more complex than QED. There is just one kind of photon, but eight different kinds of gluon. QCD features an entirely new "colour" charge, which comes in three flavours (and three anti-flavours) rather than the two (positive and negative) of QCD. This colour charge is possessed by quarks and gluons, and it influences how they interact with each other. This is another significant departure from QED. Photons are electrically neutral and incapable of interacting with one another-this is why when you look at sun the light from arrives at your eye without any interference from the light from other objects in your environment that you can also see. The QCD colour charge is far more permissive: it allows quarks to interact with other quarks, quarks to interact with gluons, and gluons to interact with themselves.

One of the more surprising features of QCD concerns the masses of protons and neutrons. In the case of the proton (the neutron is very similar) its basic constituents

\footnotetext{
39 It was quite a feat to succeed in probing experimentally this very tiny realm: a typical sugar lump contains something in the order of $6 \times 10^{23}$ atoms, and atomic nuclei occupy a volume around 100,000 times less than atoms themselves.
} 
are quarks and gluons, and gluons are entirely massless. The quarks do have mass, but not very much. In the preferred units of particle physics, the masses of a proton's three quarks add up to a total of $9.8 \mathrm{MeV} / \mathrm{c}^{2}$. The proton itself weighs $938.27 \mathrm{MeV} /$ $\mathrm{c}^{2}$. There is obviously a considerable discrepancy between these two figures: the total mass of a proton's constituent quarks amounts to less than $2 \%$ of the mass of a proton! Since gluons are entirely massless, they can't help. So where does the remainder of a proton's mass come from?

QCD does provide an answer, but it's a complex one and the full details are still being worked out. However, if the current picture is along the right lines it is one that panpsychists should not ignore.

It turns out that the major contributor to ordinary mass is the gluon field that binds quarks so strongly. Another contribution comes from the large numbers of transient virtual "sea quarks" continually coalescing from the ever-fluctuating quantum fields-forming the so-called quark condensate. The fast-moving quarks also possess a considerable amount of kinetic energy and momentum. Getting to grips with this complexity is not easy, but thanks to improved algorithms and more powerful machines computer simulations of nucleon interiors are steadily improving. Co-discoverer of QCD Frank Wilczek summarizes thus: "Indeed most ( 95\%) of the mass of protons and neutrons, and thus of ordinary matter, is due to the backreaction of color gluon fields resisting accelerated motion of the quarks and gluons inside" (Wilczek, 2012, p. 1026) ${ }^{40}$ A striking illustration of the degree to which the mass of nucleons (and hence ordinary matter) is independent of the masses of their constituent quarks comes from "QCD-lite", a simplified form of the theory which is more computationally tractable. In QCD-lite the mass of the constituent quarks is set to zero (this is one of the simplifications) but most of the predictive successes of ordinary QCD are replicated and the protons and neutrons still have $90 \%$ of their actual mass - a mass that derives entirely from processes triggered in gluon fields.

When the discovery of the Higgs boson was announced at CERN in 2012 one encountered press reports where it was suggested that the Higgs was responsible for the existence of mass, and this had now been explained. As will now be clear, this is somewhat misleading. It true that the Higgs boson provided evidence that the Higgs field exists, and in the Standard Model the latter is responsible for the mass of the fundamental particles - the quarks principally. But as we have just seen, quarks themselves are surprisingly lightweight, and contribute only a small percentage of the mass of ordinary matter. The rest comes from high-energy gluon field processes that take place within the interiors of protons and neutrons.

Since the gluon field is massless and timeless, it looks very much as though, on our current understanding of things, a sizable proportion of the mass of ordinary material things derives from processes which are themselves massless and therefore

\footnotetext{
40 According to a more recent simulation of this kind (Yang et al. 2018), 9\% of proton mass derives from the quark condensate, $\sim 32 \%$ from energy possessed by quarks, $\sim 37 \%$ from processes in the gluon field and $\sim 23 \%$ from the so-called anomalous gluonic contribution. André Walker-Loud (2018) provides a non-technical overview of this work, as does Ethan Siegel (2019). Wilczek $(2005,2008)$ and Baggott (2017) are also useful.
} 
timeless. ${ }^{41}$ For reasons already outlined, if this picture is along the right lines it is poses serious problems for the panpsychist.

\section{Lessons?}

If it initially strikes us as bizarre to suppose entities such as quarks and electrons possess experiential intrinsic natures panpsychists point out that it is crucially important to appreciate that this claim is not in the least in conflict with anything that physics has to tell us about the nature of quarks or electrons. Physics is confined to discovering the structural and causal properties of the basic ingredients of the physical world. When it comes to the intrinsic natures of these ingredients- the kind of stuff that possesses these structural and causal properties-Strawson insists that we must accept that "Physics is silent-perfectly and forever silent-on this question." (2016)

So far as the aims and ambitions of most physicists is concerned this may well be true. But as we have seen, even if physics is silent in this respect it may also be the case that some of what it does say might nonetheless have significant implications for the kinds of categorical natures the physical world's elementary ingredients possess. The existence of multiple types of elementary particle in the Standard Model undermines Nagel's attempt to generalize from our neural processes to the wider universe. I have also suggested it is possible for empirical findings to cast doubt on the claim that the physical realm is categorically homogeneous. If the causal properties of certain types of basic entities-e.g. fermions and bosons-are very different, then given certain assumptions this is strong evidence that they possess fundamentally different categorical natures. In which case if fermions are conscious, bosons might not be. ${ }^{42}$ Moreover, as we have seen latterly, the temporal properties of massless particles and fields might be incompatible with their possessing experiential inner natures - a problem which may not be confined to light and electromagnetism.

In a different vein-but moving in the same general direction-in a recent article Adam Barrett has considered whether the various quantum fields found in the Standard Model are all equally well-suited to supporting consciousness of

\footnotetext{
${ }^{41}$ Frank Wilczek is fond of summarizing these results by suggesting that for nucleons at least QCD delivers "mass without mass" (e.g. Wilczek 1999, 2012). Perhaps it also delivers mass without time.

42 Another important strand to the story which I have not been able to pursue here is the long-running attempt to unify the different components of the Standard Model-specifically the electro-weak and strong theories. While less ambitious than quantum gravity theories these attempted unifications are nonetheless important and relevant to our theme. If (for example) it were to turn out that the forces of the Standard Model were all really a single force manifesting itself in different ways, then panpsychists could take this as evidence that everything physical does have the same kind of intrinsic nature. However, the prospects for the unification programs currently do not look good. The failure to detect the predicted proton decay has ruled out one promising class of theories, whereas another approach - supersymmetryhas been undermined by the non-appearance (thus far) of any of the predicted "super-partner" particles at the Large Hadron Collider. For more on these results and the resulting crisis in particle physics see Wolchover (2016), Falkowski (2017) and Hossenfelder (2018). See Maudlin (1996) for an illuminating critical overview of the unification program.
} 
the complex kind humans enjoy. He suggests there are some clear lessons: "the strong and weak forces don't propagate over distances much larger than the width of the nucleus of an atom, and gravity alone cannot generate complex structures by virtue of being solely attractive; in contrast, the electromagnetic field can propagate over macroscopic scales, is both repulsive and attractive, and is fundamentally what enables non-trivial chemistry and biology" (Barrett, 2014, p. 4). There is more to be said here, obviously, but for present purposes we have here an interesting example of a further way in which physics may not be silent: it may have something to say about the kinds of consciousness different sorts of physical system can possess.

If the fundamental physical sciences are not necessarily completely silent with regard to the plausibility or otherwise of panpsychism then panpsychists cannot afford to completely ignore these sciences when defending and elaborating their position. They need to engage with them, and give careful thought to potential problems arising from relevant the sciences, and find ways of solving or circumventing these problems — or modifying their own position, and if need be retreating from full panpsychism to a weaker position. This engagement may well lead to an investigation into the relevance of the different solutions to the quantum measurement problem and the competing ontological interpretations of quantum field theory. I have made no attempt to assess the impact of these here, but they may well prove to be important. Another issue I have not been able to consider is the impact of the different scales that are encountered in recent physics. The events that are routinely detected and measured in particle physics experiments are many (many) orders of magnitude smaller and briefer than anything we encounter or experience in everyday life. Explaining how it is possible for such small-scale events to possess anything remotely resembling our consciousness is another challenge for the panpsychist.

If developments in physics can leave panpsychists vulnerable in areas they thought secure, the fact that physics can have implications concerning the intrinsic natures of material things opens up interesting and potentially fruitful new lines of enquiry for panpsychists to pursue. Some issues concern the nature of consciousness itself. It is often assumed that consciousness is necessarily temporal, but is this really the case? If it isn't the timelessness of certain parts of the material realm pose panpsychists less of a problem. It is even possible that panpsychists will be able to exert some influence on the development of physics itself. As the case of electromagnetic radiation illustrates, at the very least it can provide an impetus to reevaluate theoretical approaches which might not otherwise receive the attention they deserve.

Needless to say, all this applies both to widely accepted current theories in physics such as the Standard Model, and the theories which will one day supplant them. The long-standing and as yet unresolved task of reconciling quantum mechanics with general relativity has proven so difficult that many physicists suspect that a successful theory of quantum gravity might have to differ in fundamental ways from current theories. We will have to wait and see whether the new theoretical frameworks which emerge will prove more or less problematic for panpsychists. But of 
one thing we can I think be reasonably confident: when these matters come to be considered, physics will not be a totally silent partner. ${ }^{43}$

Open Access This article is licensed under a Creative Commons Attribution 4.0 International License, which permits use, sharing, adaptation, distribution and reproduction in any medium or format, as long as you give appropriate credit to the original author(s) and the source, provide a link to the Creative Commons licence, and indicate if changes were made. The images or other third party material in this article are included in the article's Creative Commons licence, unless indicated otherwise in a credit line to the material. If material is not included in the article's Creative Commons licence and your intended use is not permitted by statutory regulation or exceeds the permitted use, you will need to obtain permission directly from the copyright holder. To view a copy of this licence, visit http://creativecommons.org/licen ses/by/4.0/.

\section{References}

Baggott, J. (2017). Mass: The quest to understand matter from Greek atoms to quantum fields. Oxford University Press.

Barrett, A. (2014). An integration of integrated information theory with fundamental physics. Frontiers in Psychology, 63, 5.

Brüntrup, G. (2016). Emergent panpsychism. In G. Brüntrup \& L. Jaskolla (Eds.), Panpsychism. Oxford University Press.

Carroll, S. (2012). The particle at the end of the universe. Oneworld Publications.

Coopersmith, J. (2015). Energy, the subtle concept: The discovery of Fenyman's blocks from Leibniz to Einstein (2nd ed.). Oxford University Press.

Cramer, J. G. (1986). The transactional interpretation of quantum mechanics. Reviews of Modern Physics, 58(3), 647-688.

Cramer, J. G. (2016). The quantum handshake: Entanglement, Nonlocality and Transaction. Springer Media.

Dainton, B. (2014). The phenomenal continuum. In D. Lloyd \& V. Arstila (Eds.), Subjective time: The philosophy, psychology and neuroscience of temporality. MIT Press.

Dainton, B. (2017). Temporal consciousness. Stanford Encyclopedia of Philosophy. https://plato.stanford. edu/entries/consciousness-temporal/

Dalton, J. (2010). A new system of chemical philosophy. Cambride University Press.

Davies, P. C. W. (1971). Extension of Wheeler-Feynman quantum theory to the relativistic domain I. Scattering processes. Journal of Physics A: General Physics, 6, 836-845.

Davies, P. C. W. (1972). Extension of Wheeler-Feynman quantum theory to the relativistic domain II. Emission processes. Journal of Physics A: General Physics, 5, 1025-1036.

Drake, D. (1925). Mind and its place in nature. Macmillan.

Einstein, A. (1949). Autobiographical notes. In P. A. Schilpp (Ed.), Albert Einstein-philosopher scientist (pp. 1-95). Tudor Publishing.

Falkowski, A. (2017). Higgs on the moon. Inference, International Review of Science (3)1. https://infer ence-review.com/article/higgs-on-the-moon

Feng, J. (2010). Dark matter candidates from particle physics and methods of detection. Annual Review of Astronomy and Astrophysics, 48, 495-545.

Feynman, R. (1964). Lectures on physics (Vol. 1). Addison-Wesley.

Foster, J. (1982). The case for idealism. Routledge.

Foster, J. (2008). A world for us: The case for phenomenalist idealism. Oxford University Press.

\footnotetext{
${ }^{43}$ My thanks to two anonymous referees, David Chalmers, Milan Ćirković, Elie During, Ian Dunbar, Richard Gaskin, Philip Goff, Mike Miller, Mike Pelczar, Howard Robinson, Galen Strawson, and audiences at Budapest, Cambridge, Toulouse and The Second Hermann Minkowski Meeting on the Foundations of Spacetime Physics.
} 
Fraser, D. (2009). Quantum field theory: Underdetermination, inconsistency, and idealization. Philosophy of Science, 76, 536-567.

Fraser, D. (2011). How to take particle physics seriously: A further defence of axiomatic quantum field theory. Studies in the History and Philosophy of Modern Physics, 42, 126-135.

Freeman, A. (ed.) (2006). Consciousness and its place in nature: Does physicalism entail panpsychism? Imprint Academic.

Gribbin, J. (1995). Schrödinger's Kittens and the search for reality. Little Brown.

Goff, P. (2017a). Consciousness and fundamental reality. Oxford University Press.

Goff, P. (2017b). Panpsychism. Stanford Encyclopedia of Philosophy. https://plato.stanford.edu/entries/ panpsychism/

Goff, P. (2020). Revelation, consciousness+ and the phenomenal powers view. Topoi, 39, 1089-1092.

Heisenberg, W. (1958). Physics and philosophy. Harper and Brothers.

Hossenfelder, S. (2018). Lost in math: How beauty leads physics astray. Basic Books.

Howell, R. (2015). The Russellian monists problems with mental causation. Philosophical Quarterly, 65(258), 22-39.

Hoyle, F., \& Narlikar, J. V. (1995). Cosmology and action-at-a-distance electrodynamics. Reviews of Modern Physics, 67(1), 113-155.

Kastrup, B. (2019). The idea of the world. iff Books.

Kastner, R. E. (2012a). The transactional interpretation of quantum mechanics: The reality of possibility. Cambridge University Press.

Kastner, R. E. (2012b). The new possibilist transactional interpretation and relativity. Foundations of Physics, 42, 1094-1113.

Kastner, R. E. (2016). The transactional interpretation and its evolution into the 21st century: An overview. arXiv:1608.00660.

Kuhlman, M. (2020). Quantum field theory. https://plato.stanford.edu/entries/quantum-field-theory/.

Lange, M. (2001). The most famous equation. The Journal of Philosophy, 98(5), 219-238.

Lange, M. (2002). An introduction to the philosophy of physics: Locality, Fields, energy, and mass (p. 2002). Blackwell.

Maudlin, T. (1996). Unification in physics. Journal of Philosophy, 93(3), 129-144.

Mørch, H. H. (2018a). Does dispositionalism entail panpsychism? Topoi, 39.

Mørch, H. H. (2018b). The evolutionary argument for phenomenal powers. Philosophical Perspectives, 31(1).

Mørch, H. H. (2019). The argument for panpsychism from experience of causation. In W. Seager (Ed.), The Routledge handbook of panpsychism. Routledge.

Nagel, T. (1979). Panpsychism. Cambridge University Press.

Nagel, T. (1986). The view from nowhere. Oxford University Press.

Patil, Y. S., Chakram, S., \& Venglattore, M. (2015). Measurement-induced localization of an ultracold lattice gas. Physical Review Letters, 115(14), 115.140402-1-140402-5.

Penrose, R. (2010). Cycles of time. Bodley Head.

Rickles, D. (2016). The philosophy of physics. Polity.

Russell, B. (1927). The analysis of matter (pp. 55-56). Kegan Paul, Trench and Trubner.

Russell, B. (1928). Review of Sir Arthur Eddington. The Nature of the Physical World. In J. G. Slater (Ed.), (1996) The collected papers of Bertrand Russell volume 10. Routledge.

Schneider, S. (2018). Spacetime emergence, panpsychism and the nature of consciousness. https://blogs. scientificamerican.com/observations/spacetime-emergence-panpsychism-and-the-nature-of-consc iousness/.

Seager, W. (Ed.). (2020). The Routledge handbook of panpsychism. Routledge.

Siegel, E. (2019). Ask ethan: Where does a proton's mass come from? Forbes. https://www.forbes.com/ sites/startswithabang/2019/04/27/ask-ethan-where-does-a-protons-mass-come-from/?sh=75f25 $6 \mathrm{c} 116 \mathrm{be}$

Silberstein, M., Stuckey, W. M., \& McDevitt, T. (2018). Beyond the dynamical universe: Unifying block universe physics and time as experienced. Oxford University Press.

Skinner, B. (2015). A child's picture-book introduction to quantum field theory. https://www.ribbonfarm. com/2015/08/20/qft/

Strassler, M. (2012). Matter and energy: a false dichotomy. Of Particular Significance. https://profmattst rassler.com/articles-and-posts/particle-physics-basics/mass-energy-matter-etc/matter-and-energy-afalse-dichotomy/

Strawson, G. (1987). Realism and causation. The Philosophical Quarterly, 37(148), 253-277. 
Strawson, G. (2003). Real materialism. In L. Anthony \& N. Hornstein (Eds.), Chomsky and his critics (pp. 49-88). Blackwell.

Strawson, G. (2006a). Realistic monism: Why physicalism entails panpsychism. In Freeman (2006) (pp. 3-31).

Strawson, G. (2006b). Panpsychism? Reply to commentators with a celebration of Descartes. In Freeman (2006) (pp. 184-280).

Strawson, G. (2008a). The identity of the categorical and dispositional. Analysis, 68(4), 271-282.

Strawson, G. (2008b). Selves: An essay in revisionary metaphysics. Oxford University Press.

Strawson, G. (2013). Real naturalism. London Review of Books, 26 Sept.

Strawson, G. (2016). Consciousness isn't a mystery. It's matter. New York Times.

Strawson, G. (2017a). Mind and being: The primacy of panpsychism. In G. Gruntrup \& L. Jaskolla (Eds.), Panpsychism: contemporary perspectives (pp. 75-112). Oxford University Press.

Strawson, G. (2017b). Physicalist panpsychism. In S. Schneider \& M. Velmans (Eds.), Blackwell companion to consciousness (2nd ed., pp. 374-390). Wiley-Blackwell.

Strawson, G. (2017c). Conceivability and the silence of physics. Journal of Consciousness Studies, 24(11-12), 167-192.

Strawson, G. (2018). The consciousness deniers. NYRB.

Strawson, G. (2020). What does "physical" mean? A prolegomenon to physicalist panpsychism. In Seager (Ed.) (pp. 317-339).

Walker-Loud, A. (2018). Dissecting the mass of a proton. Physics, 11(118). https://physics.aps.org/artic les/v11/118

Wallace, D. (2011). Taking particle physics seriously: A critique of the algebraic approach to quantum field theories. Studies in the History and Philosophy of Modern Physics, 42, 116-125.

Wesley, D. H. \& Wheeler, J. A. (1972). Towards an action-at-a-distance concept of spacetime. In A. Ashtekar et al. (Eds.), Revisiting the foundations of relativistic physics: Festschrift in Honor of John Stachel. Boston studies in the philosophy and history of science (pp. 421-436). Kluwer Academic Publishers.

Wilczek, F. (1999). Mass without mass. Physics Today 52N11, 13.

Wilczek, F. (2005). Nobel lecture: Asymptotic freedom: From paradox to paradigm. Reviews of Modern Physics, 77.

Wilczek, F. (2008). The lightness of being. Penguin.

Wilczek, F. (2012). Origins of mass. Central European Journal of Physics, 10(5), 1021-1037.

Wheeler, J. A. (with Kenneth Ford). (1998). Geons, black holes and quantum foam. Norton.

Wheeler, J. A., \& Feynman, R. P. (1945). Interaction with the absorber as the mechanism of radiation. Reviews of Modern Physics, 17(2-3), 157-181.

Wheeler, J. A., \& Feynman, R. P. (1949). Classical electrodynamics in terms of direct interparticle action. Reviews of Modern Physics, 21(3), 425-433.

Wilson, A. (2018). Skow on the passage of time. Analysis, 78(1), 117-128.

Wolchover, N. (2016) What no new particles means for physics. Quanta.

Yang, Y. B., et al. (2018). Proton mass decomposition from the QCD energy momentum tensor. Physical Review Letters, 121, 212001.

Publisher's Note Springer Nature remains neutral with regard to jurisdictional claims in published maps and institutional affiliations. 